\title{
AN ULTRA FAST AGILE LOCAL OSCILLATOR FOR CUEUING CHANNELIZED RECEIVERS
}

\author{
ALADIN H. ASSISI ${ }^{1}$
}

\begin{abstract}
An ultra fast frequency agile local oscillator with high spectral purity is presented. It can be used in a queuing channelized receiver to generate the required local oscillator frequency for each newly received pulse within the pulse duration. It can also be used as a local oscillator in pulse-to-pulse frequency agile radars with high pulse repetition frequencies. The time necessary to change the oscillator frequency is that of the output electronic switch. The phase noise and the frequency stability are those of the crystal oscillator. The spectral purity is guaranteed by the proper filter design.
\end{abstract}

\section{KEY WORDS}

Channelized receiver, cueing receiver, local oscillator, step recovery diode.

\section{NOMEMNCLATURE}

SPLT Single Pole L Through electronic switch

SRD Step Recovery Diode

\section{INTRODUCTION}

Substantially increasing demands are being placed on receivers for communications and radar signal reception applications in the electromagnetic frequency range from $2 \mathrm{MHz}$ to $300 \mathrm{GHz}$. These requirements are driven by the nearly exponentially increasing number of signals per unit time that need to be intercepted, sorted, and, in some cases, classified [1]. In parallel channelized

\footnotetext{
${ }^{1}$ Ass. Prof. , Electronic Warfare Engineering Department; MTC, Cairo
} 
receivers, the full electromagnetic frequency signal bandwidth of interest is divided into many contiguous segments (Fig. 1); each segment is responsive only to a small portion of the full bandwidth of the electromagnetic environment. Each parallel signal processing channel of the receiver is responsive only to one of many separate channel bands of the full segmented frequency bandwidth. Because the parallel channels are independent, several time-coincident signals can be intercepted simultaneously without loss of information [1]. A channelized receiver is the most preferable architecture for electronic warfare applications, since it combines the high selectivity and sensitivity of a selective receiver, together with the high intercept probability of a wide-open receiver. A queuing channelized receiver is the most preferable architecture for EW receiver channelization, since it combines the high frequency resolution of a fully channelized receiver with the low hardware count of a band-folded channelized receiver. At the same time, it does not have the ambiguity and sensitivity problems of a band-folded channelized receiver [2]. The purpose of a queuing receiver is to detect the presence of multiple emitters in a wide band of interest and to pass this information to other receivers with narrower bandwidth and greater selectivity [3]. A single stage of queuing channelization is shown in Fig. 2.

\section{DESCRIPTION OF THE PROBLEM}

A cueing-channelized receiver consists of $L$ parallel receivers, each covering a certain sub-band (i) of the total frequency band [2]. A channelized front end determines the sub-band to which a received RF pulse belongs and selects the suitable local oscillator frequency to down-convert this RF pulse to the first intermediate frequency band of the system. The process is repeated at the subband level, dividing it into $\mathrm{M}$ channels and on each channel dividing it into $\mathrm{N}$ subchannels. Such a system needs ultra fast agile local oscillators capable of switching to the required frequency within a short portion of the received pulse duration. Given that the pulse durations of modern radars may go down to 100 [ns]; it is evident that the whole process of channel determination and LO frequency selection must be done within a few tens of nanoseconds. This is a stringent design requirement to be fulfilled.

\section{COMPARING DIFFERENT ALTERNATIVE SOLUTIONS}

In order to get the correct LO frequency within a very short time, we may use a frequency synthesizer; i.e. a PLL with real-time controllable division ratio (Fig. 3). A closed loop synthesizer has its inherent time delay, which is much longer than the available time. Another candidate solution is an open loop Direct Digital synthesizer (DDS). The state of the art of DDS together with its control circuitry does not fulfill the required tuning speed [11]. The simplest alternative logical solution would be to build a set of $L$ different oscillators; each pre-tuned to one of 
the required frequencies and select one of them according to the received signal channel. This is a rather costly solution.

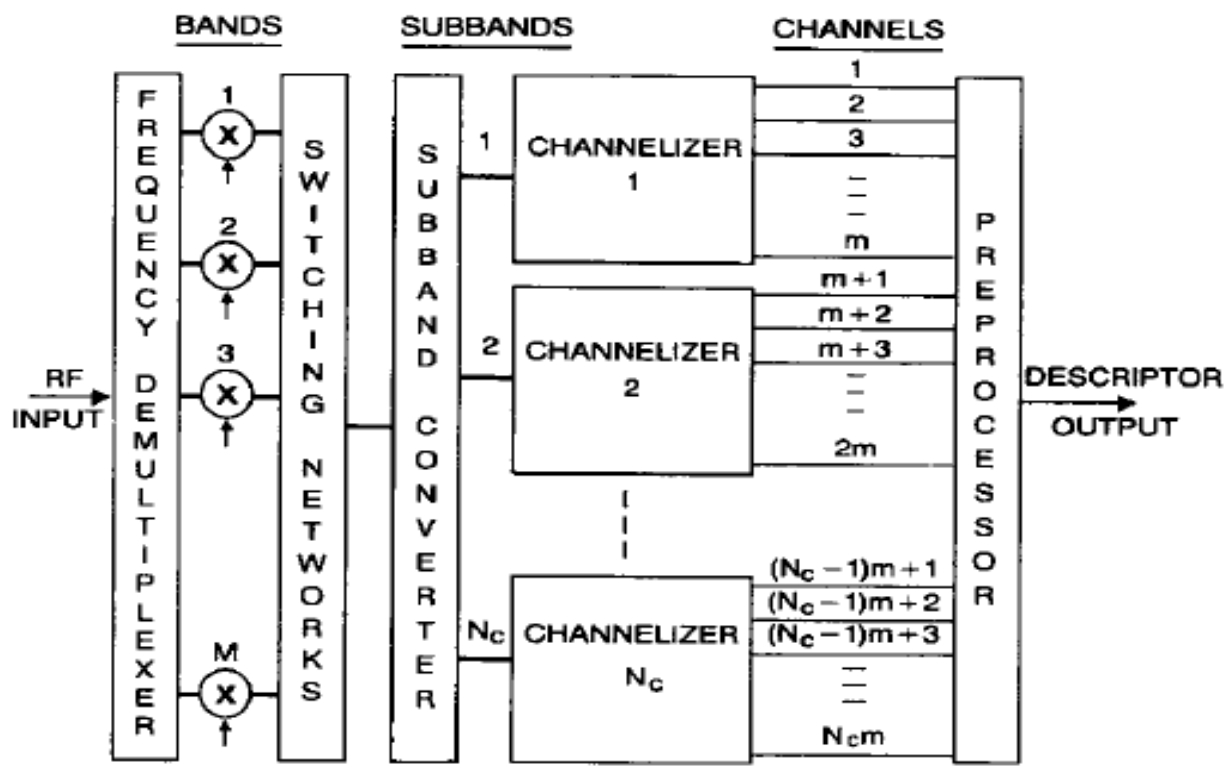

Fig.1. Three-level channelized receiver architecture having $M$ channelizers and $N$ channels for each channelizer [1].

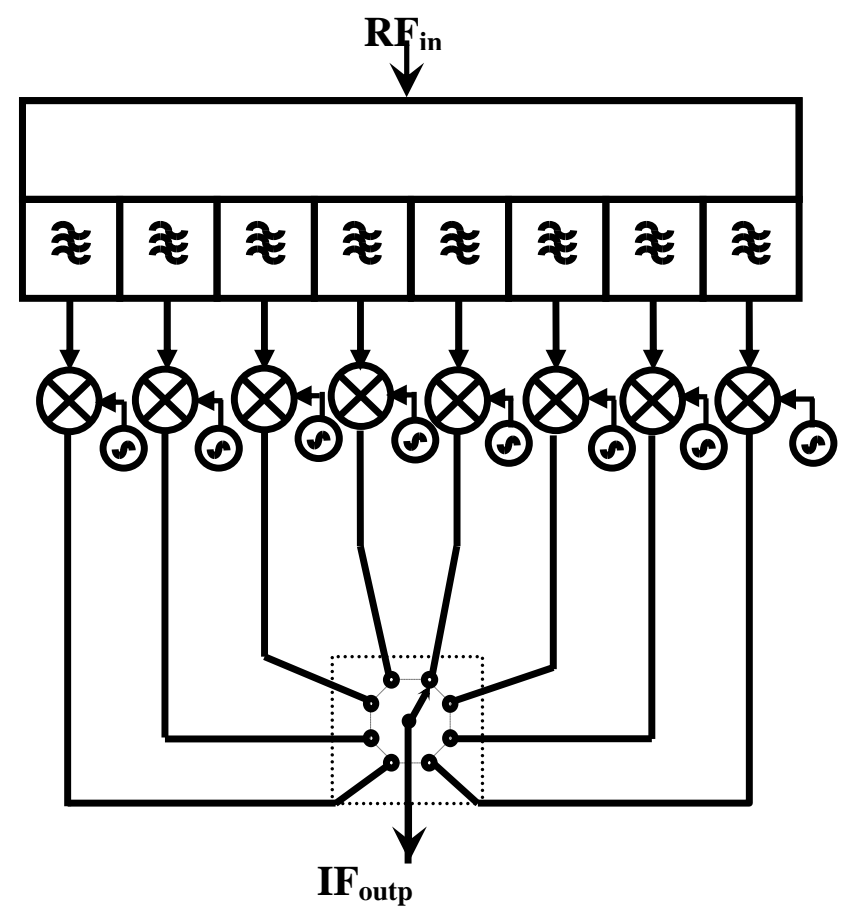

Fig.2. A single stage of channelization in an 8-channel cueing receiver 


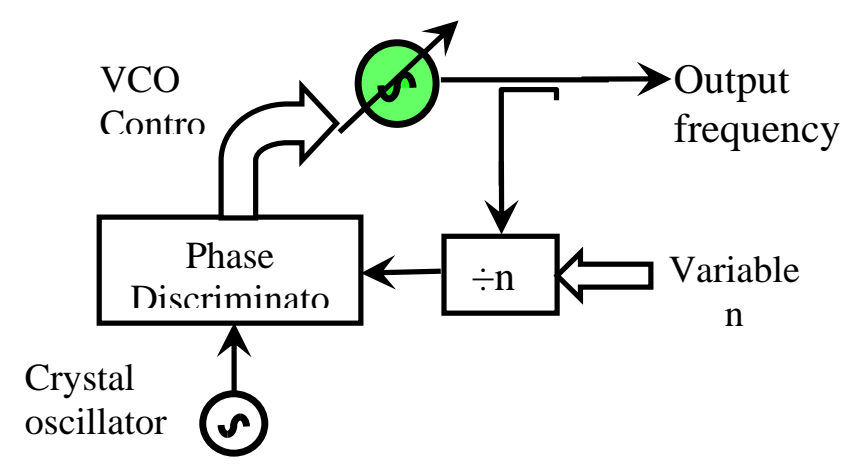

Fig. 3. A PLL with real-time controllable division ratio

A more economic approach is to use a step recovery diode comb generator with a set of $L$ selective filters, each tuned to one of the comb output frequencies. A switched filter bank with 2 SPLT switches at its input and output forms, together with the comb generator, an attractive candidate solution.

A theoretical analysis of the step-recovery diode performance when loaded with a band pass filter shows that the filter reflects the un-tuned harmonics back to the diode where they mix to form additional power at the tuned frequency [5].

In order to validate this important phenomenon, the measurement set-up shown in Fig. 4. was implemented by our team. We put a dual directional coupler at the output of a step recovery diode. An SPDT electronic switch was used to switch the coupler output between a $2.5 \mathrm{GHz}$ band-pass filter and a $50 \mathrm{Ohm}$ load. A clear $6 \mathrm{~dB}$ increase in the pass-band frequency power was measured at the direct wave output when the SPDT was switched to position 1. This emphasizes the spectral re-distribution suggested by ref. [5]. Such a power re-distribution among different frequency components at the SRD output causes a ringing process after the connection of the filter [6,7]. This ringing takes time after each filter connection until the SRD gives a stable output spectrum. Although it was difficult to measure this transient time without a real-time spectrum analyzer, we are sure that such a transient process increases the time necessary for output readiness after each filter switching.

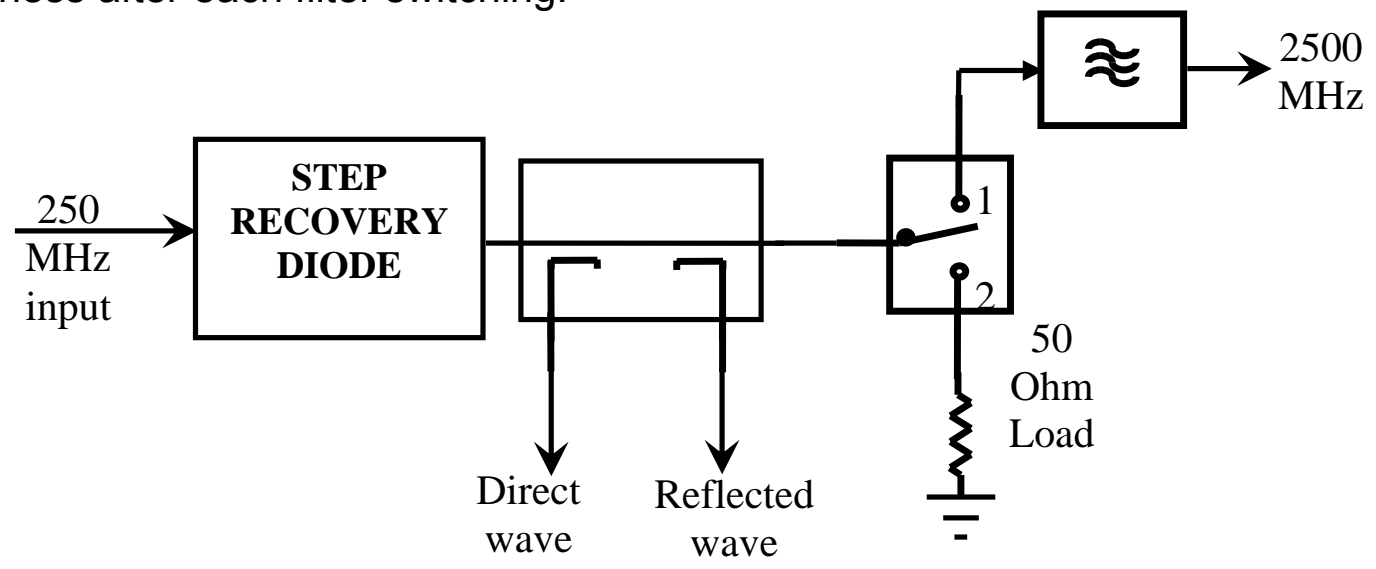

Fig. 4. Measurement set-up for spectral re-distribution testing 

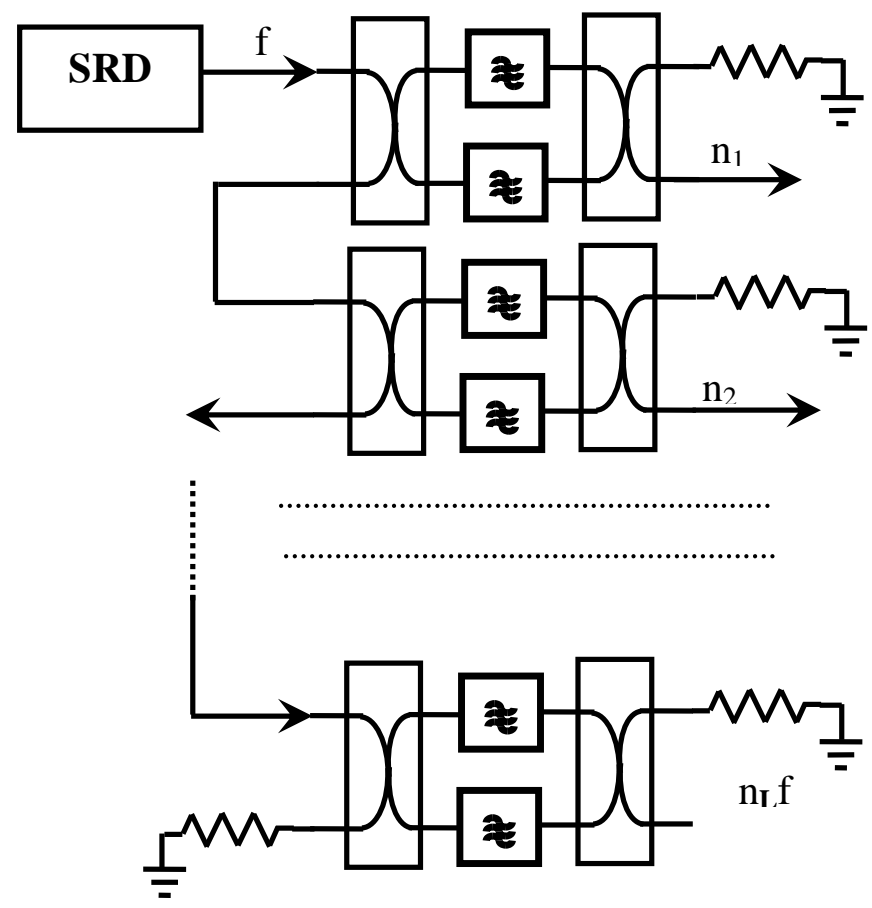

Fig. 5. Frequency multiplexing using balanced filters

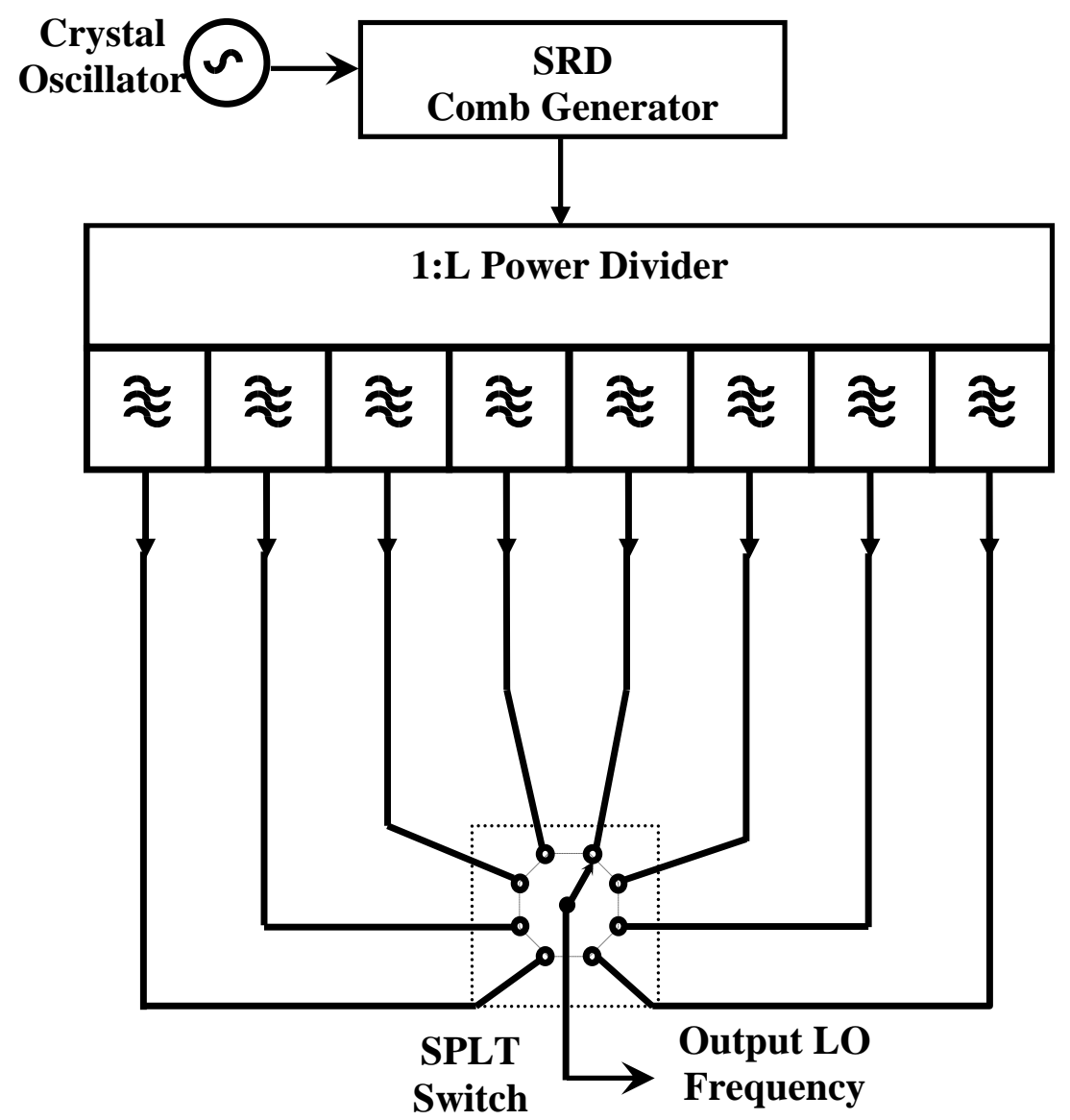

Fig. 6. The proposed solution for agile local oscillator 
The next two solutions are either to use a set of balanced filters (Fig. 5), each composed of two $90^{\circ}$ hybrid junctions and two identical band pass filters as described in [3], or to use a power splitter followed by $L$ band pass filters and switch among the outputs of those filters.

Although the balanced filters solution guarantees controlled reflection performance; two difficulties are there: The first is the difficulty to build a wideband hybrid junction that covers the complete desired frequency band. The second is the doubled number of filters and hybrids, which means an excessive hardware cost. In spite of its increased insertion loss, we have adopted the last solution (Fig. 6); since it gives L continuously existing output frequencies among which we can easily and quickly switch by using only $L$ different filters, one $1: L$ power splitter and a fast absorptive SPLT switch at the output. No ringing will occur since the switch is located after the parallel filter bank.

\section{DESIGN OF SELECTIVE FILTERS}

Each filter $i$ has to give $S_{11}<-20[d B]$ at fi and $S_{21}<-50[d B]$ both at $f_{i-1}$ and $f_{i+1}$. It has to give a monotonic decreasing $S_{21}$ function with frequency deviation If $-\mathrm{f}_{\mathrm{i}}$. Since microstrip filters show parasitic pass bands at higher frequencies [8], the S21 should be minimized within those parasitic pass bands. It should not exceed $-30[\mathrm{~dB}]$.

We have designed and implemented 8 different filters, each fulfilling better performance than this specification. We have selected a modified combline filter design; since it gives a controlled performance with much shorter resonators due to the capacitive loading [8]. A combline bandpass filter consists of mutually coupled resonators which are physically less than $\lambda / 4$ long, grounded at one end and capacitively loaded at the other. Resonator line lengths between $30^{\circ}$ and $45^{\circ}$ result in a compact structure with excellent stop-band performance [9].

Attention has been paid to minimize parasitic pass bands up to 18 [GHz] to prevent any spurious harmonics of the comb generator from the output. However, using band-limited smb connectors guarantees very good suppression of higher order harminics. The designs have been optimized through subsequent stages of linear, electromagnetic and combined simulations using the capabilities of the GENESYS CAD package. The design of one of these filters is shown in Fig. 7. It can be noticed how the above-mentioned specifications are fulfilled. 


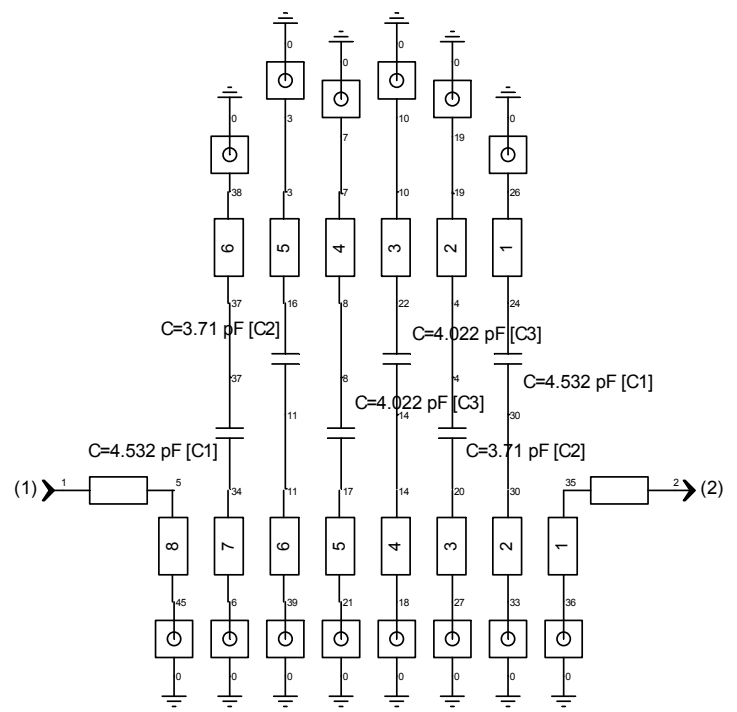

a. Schematic Design

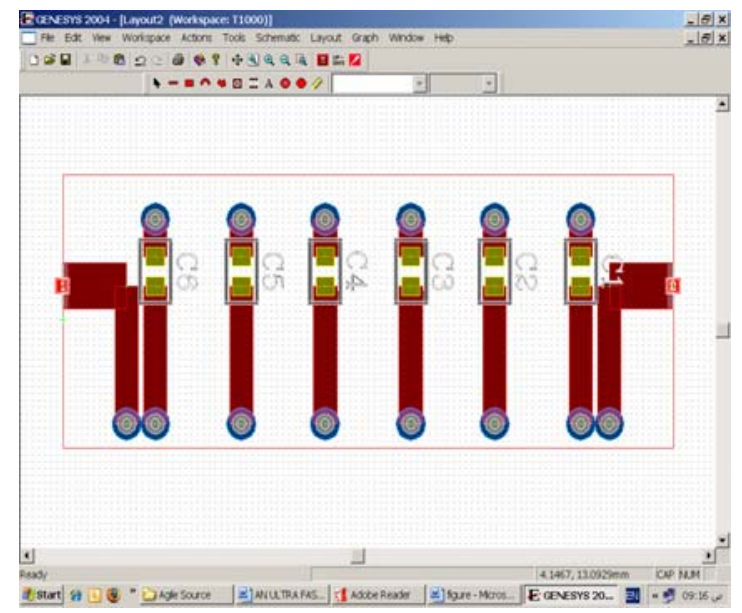

b. Microstrip Layout Design
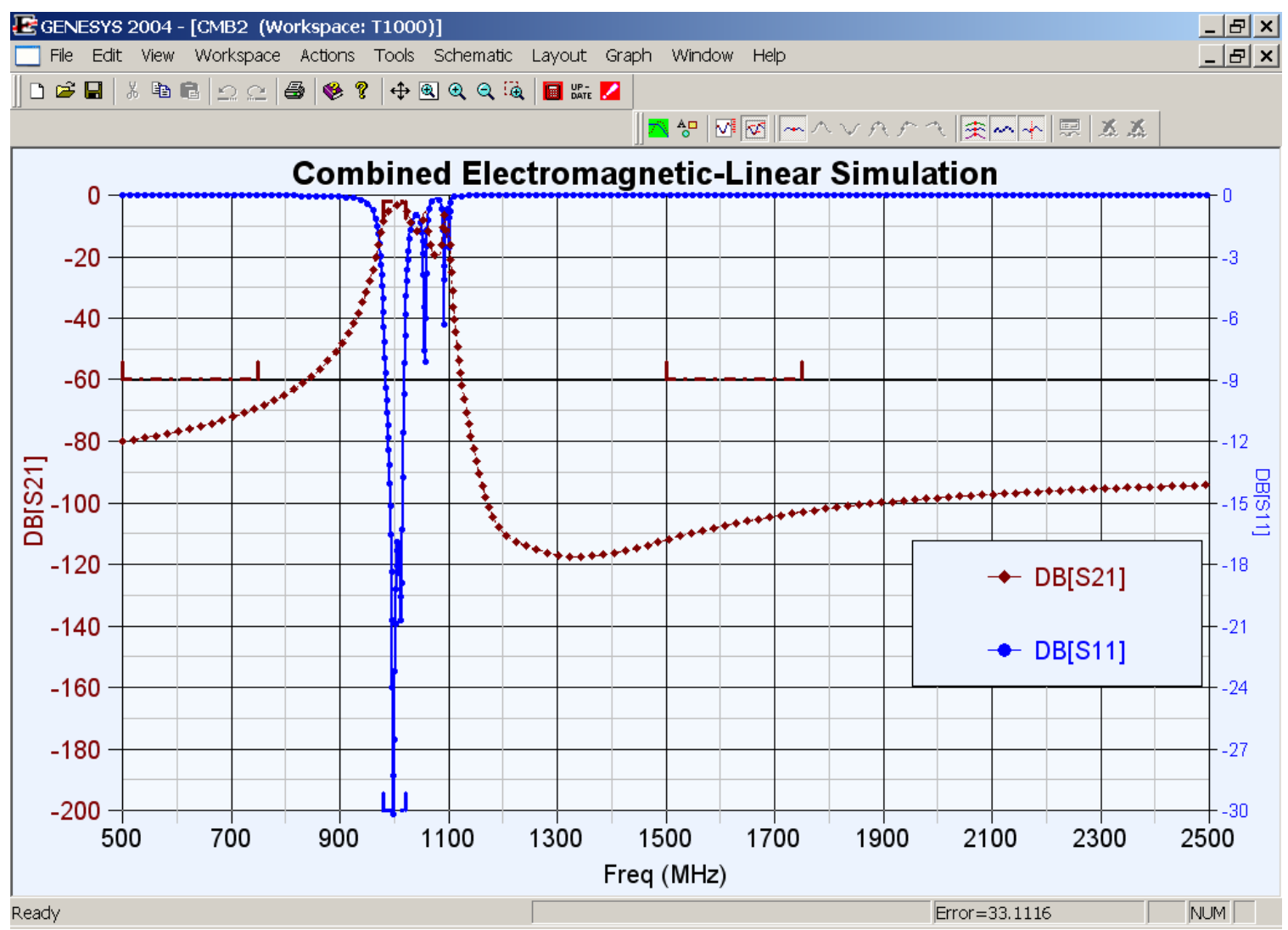

\section{Combined Electromagnetic-Linear Simulation}

Fig.7. Design of a $1 \mathrm{GHz}$ selective filter 


\section{DESIGN OF THE WIDE-BAND POWER SPLITTER}

The power splitter has to cover an extended frequency band (1 to $3 \mathrm{GHz}$ ) in order to cover 8 subsequent outputs of the $250 \mathrm{MHz}$ comb generator. The most important design parameters are input and output matching $\left(S_{\mathrm{jj}}\right.$ must be less than $-10 \mathrm{~dB}$ for $\mathrm{j}=1$ to 9 ) and the mutual coupling between each two outputs must be minimized $\left(S_{\mathrm{jk}}<-10 \mathrm{~dB}\right)$. This mutual isolation will be added to the SPNT isolation at the output. Linear, electromagnetic and combined simulations of the Wilkinson 8-way power splitter we have designed have shown compliance to this specification. A sample of the simulation results is shown in Fig. 8.

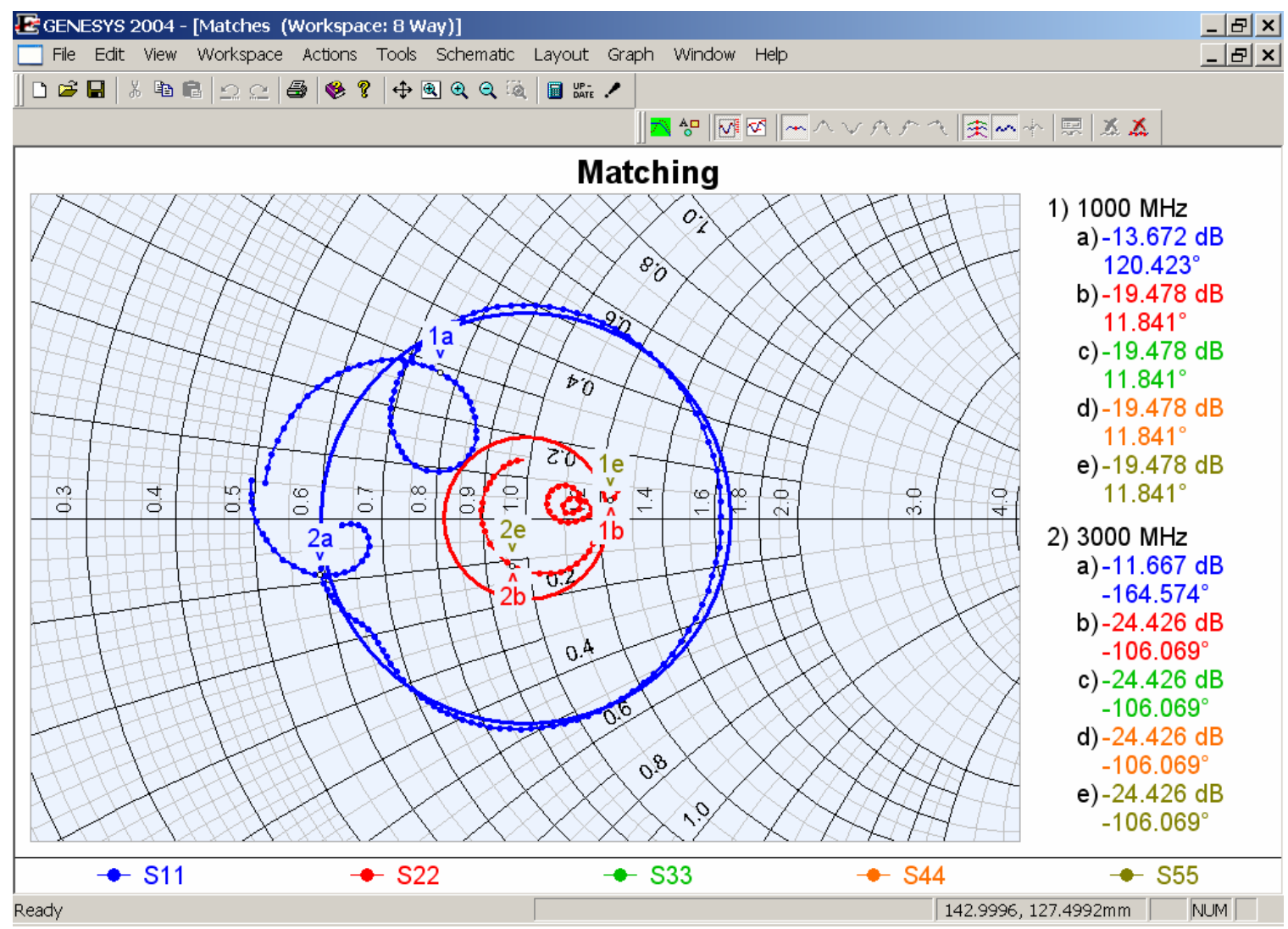

Fig.8 Matching performance of the 1-3 GHz wide-band power divider

\section{PERFORMANCE SIMULATION}

A linear simulation was done on the power splitter loaded with the eight filters. The whole assembly was optimized to give a controlled performance at the different 8 output frequencies satisfying the design requirements. $A 3 \mathrm{~dB}$ resistive attenuator was inserted at the splitter input to enhance input matching. The linear simulation of the LO assembly shows that each output has a very high selectivity at a certain frequency. It has been noticed that the S11 of the system increased 
from - 10 to -6 due to the loading with the band pass filters. This loading will not harm the SRD if we consider the interpretation that the reflected frequencies will cause the SRD to re-distribute its power on the favorite frequencies of different filters.

\section{IMPLEMENTATION AND MEASUREMENT}

The layout was designed using an FR4 laminate with $\mathrm{t}=0.018 \mathrm{~mm}, \mathrm{~h}=1.5[\mathrm{~mm}]$ and $\varepsilon_{\mathrm{r}}=4.8$. The whole LO circuit board was implemented by an LPKF prototyping machine. The designed filters were implemented, tested and optimized for the required performance. The power splitter was implemented and measured with a network analyzer to verify its performance. A special microwave container was designed and implemented on a CNC machine for this module.

\section{CONCLUSION}

An ultra fast switching frequency source has been designed and implemented. It can be used as a local oscillator in a modern cueing channelized receiver. It can be also used as a local oscillator in an ultra-fast pulse-to-pulse frequency-agile radar.

\section{ACKNOWLEDGMENT}

The author would like to thank Eng. A. Salah, Eng. A. Quindeel and Eng. M. Mahmoud who implemented and tested the agile frequency source.

\section{REFERENCES}

[1] Anderson, G.W.; Webb, D.C.; Spezio, A.E.; Lee, J.N.; "Advanced Channelization For RF, Microwave, and Millimeterwave Applications", Proceedings of the IEEE, Volume 79, Issue 3, March 1991 Page(s):355 - 388.

[2] Aladdeen Assisy, "Microwave Receiving System Architectures", a PhD. Thesis, MTC, Cairo, 1993.

[3] Sollner, T.C.L.G.; Lyons, W.G.; Arsenault, D.R.; Anderson, A.C.; Seaver, M.M.; Boisvert, R.R.; Slattery, R.L.; "Superconducting cueing receiver for space experiment", IEEE Transactions on Applied Superconductivity, Volume 5, Issue 2, Part 3, Jun 1995 Page(s):2071 - 2074.

[4] James B. Tsui, Microwave Receivers with Electronic Warfare Applications,J. Wiley \& sons, 1986.

[5] Agilent Technologies, Low Cost Frequency Multipliers using Surface Mount PIN Diodes, Appplication Note No. 1054 , year 1999. 
[6] Aaron Michael Orndorff, Transceiver Design for Ultra-wideband Communications, an Msc Thesis, Virginia Polytechnic institute and State university, may 2004.

[7] George H. Stauffer Jr., SPICE up the development of a step recovery diode frequency multiplier, RF Design, June 1999.

[8] Jia-Sheng Hong, M. J. Lancaster, Microstrip Filters for RF/Microwave applications, john wiley \& sons, inc., 2001.

[9] Randall W. Rhea, "HF Filter Design ND Computer Simulation", Noble Publishing, 1994.

[10] W. M. VAN. Look, "Working Conditions of a Step-Recovery Diode Multiplier Allowing One Conduction Angle per Cycle", IEEE Proceedings, Volume 56, Issue 5, May 1968 Page(s):867 - 867.

[11] Bonfanti, A.; Amorosa, F.; Samori, C.; Lacaita, A.L.;, "A DDS-based PLL for 2.4-GHz frequency synthesis", Circuits and Systems II: IEEE Transactions on Analog and Digital Signal Processing, , Volume 50, Issue 12, Dec. 2003 Page(s):1007 - 1010. 